\title{
Performance Comparison of Transforms using Row Mean and Column Mean for Hand Gesture Recognition
}

\author{
Tanuja K. Sarode, Ph. D \\ Dept. of Computer Engineering \\ Thadomal Shahani College of Engg, \\ Mumbai, India.
}

\author{
Vaishali D. Sakpal \\ Dept. of Information Technology \\ Don Bosco Institute of Technology \\ Mumbai, India.
}

\begin{abstract}
Human computer interaction is a major issue in research industry. In order to offer a way to enable untrained users to interact with computer more easily and efficiently gesture based interface has been paid more attention. This paper presents a new approach for hand gesture recognition. An approach consists of three modules: a) Preprocessing of the image b) Feature extraction c) Pattern matching for gesture recognition. Feature extraction is based on feature vector of transformed image using Discrete Cosine Transform, Walsh Transform, Haar transform and Kekre's transform. This transforms are applied on column mean and row mean of the images and various percentage of feature vectors are generated such as $100 \%, 50 \%, 25 \%, 12.5 \%$ and $6.25 \%$. Results found to be better than existing system.
\end{abstract}

\section{General Terms}

Human Computer Interaction, Pattern recognition.

\section{Keywords}

Hand gesture recognition, Pattern Matching, feature vector.

\section{INTRODUCTION}

Hand gesture recognition is one of the important aspects in various upcoming fields like human computer interaction, virtual reality and computer vision. For hearing impaired community, the development of automatic gesture translation based natural languages (e.g. the American Sign Language; ASL) is highly expected to improve their communication means among humans. Compared with the traditional interaction approaches, such as keyboard, mouse, pen etc. vision based hand interaction is more natural and efficient. Therefore hand gesture recognition has received considerable attention.

There are number of techniques and algorithms available in the literature of hand gesture recognition. Jagdish Raheja et al [1] proposed Principle Component Analysis method for hand recognition. In his proposed system, gesture recognition is done with 10 different hand gestures which gives 90\% accuracy in proper light arrangement and is future decreasing in poor light arrangement. Yuehai Wang et al [2] used Morphological Method for hand gesture recognition. It mainly uses skin color extraction, dilation, erosion, and subtraction for hand gesture recognition. A recognition rate is $84 \%$. Yikai Fang, Kongqiao Wang et al [3] proposed hand gesture recognition using Blob and Ridge Method; hand detection is done with Adaboost to trigger tracking and recognition, Adaptive hand segmentation is executed during detection and tracking with motion and color cues, scale-space features detection is applied to find palm-like and finger-like structures. Deg-Yuan Huang et al [4] uses gabor filter and support vector machine, the principle component method is used to reduce the dimensionality of the feature space. A gesture recognition rate is 95\%. Qing Chen et al [5] used haar- like features to describe the hand posture pattern with the computation of integral image. AdaBoost learning algorithm is used to construct a strong classifier by combining a sequence of weak classifiers. This method gives accuracy above $90 \%$. Rajeshree Rokade et al [6] in his paper proposed a novel technology for hand gesture recognition which is based on thinning of segmented image. The average recognition rate is $92.13 \%$. Zhong Yang et al [7] proposed Hidden Markov Model for gesture recognition its recognition rate is $96.67 \%$ with 18 different gestures. Karishma Dixit et al [8] used a multi-class Support Vector Machine (MSVM) for training and recognition its recognition rate is $96 \%$. Archana Ghotkar et al [9] used HSV color model and Genetic algorithm for gesture recognition. Prateem Chakraborty et al [10] have given comparative study of Gradient and PCA techniques. Thus there are various techniques have been used for gesture recognition.

This paper presents hand gesture recognition techniques based on row mean and column mean of input image and implementation of feature vector of transformed image using Discrete Cosine Transform, Walsh, Haar, Kekre's transform. The result of these transformed techniques is compared with one of the existing and popular technique that is principle component analysis

\section{SYSTEM DESCRIPTION}

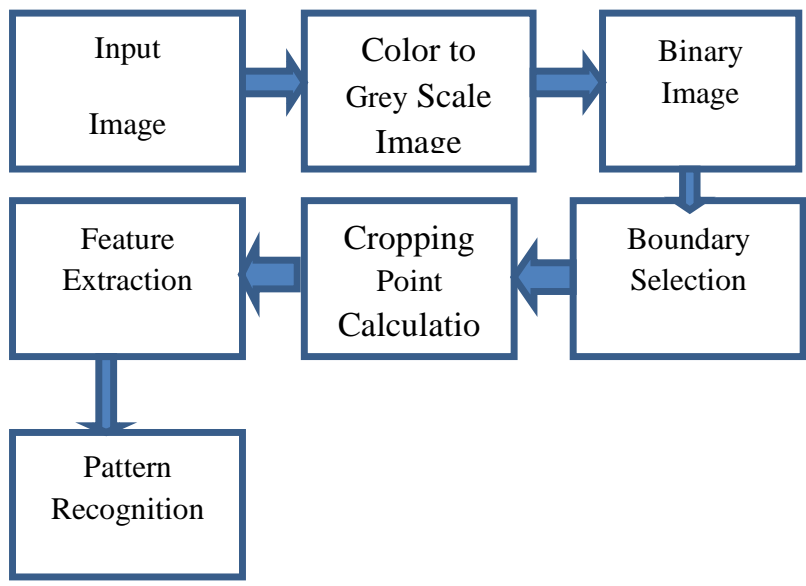

Fig.1: Block Diagram of the System

This section consists of three major components such as preprocessing, feature extraction and pattern matching. The block diagram of the system is as shown in the Fig. 1.

\subsection{Image Preprocessing}

Image Preprocessing is required to remove extra unwanted part for better result in pattern matching.

The steps for Image Preprocessing is as follows, 
1. Take the input image from stored database.

2. Convert the input image into binary image using global thresholding.

3. Extract the object of interest from binary image. This is done by searching white pixel horizontally and vertically. Occurrence of first and last white pixels are boundary points. The image is cropped according to boundary point as shown in Fig. 2 .

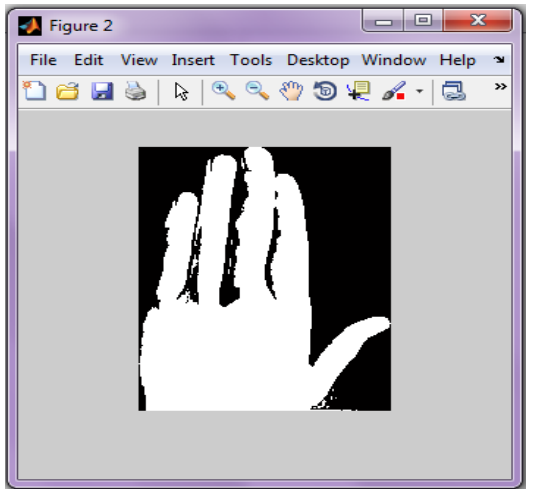

Fig. 2: Boundary Selection

4. Cropping point calculation is to remove part of a hand which is not used in gesture presentation as shown in Fig. 3. Determination of column in which maximum number of white pixels are presents. (Ex. wrist area) cropping points are between the columns of maximum and minimum number of white pixels.

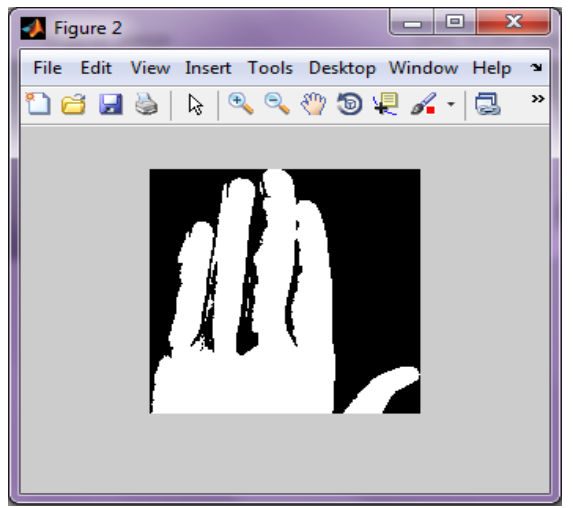

Fig. 3: Cropping Point Calculation

5. Resize the image to some standard size, in this proposed system after preprocessing the maximum size of the image is $64 \mathrm{X} 64$ therefore all the images are resize to $64 \times 64$.

\subsection{Feature Extraction}

Extraction of feature vector from images is done using different techniques. Feature vector of query image is compared with feature vector of the images in the database. euclidean distance is used to check the closeness of the query image and database image. The techniques for feature extraction is given below.

\subsubsection{Principle Component Analysis}

Principle Component Analysis [11] is used for feature extraction, algorithm is as follow:

1. Capture the image, stores it as test database. The database is stored in train database.

2. Calculate the mean of each image. Subtract the mean from each of the data dimension the mean subtracted is the average across each dimension.

3. Calculate the covariance matrix.

4. Calculate the eigenvectors and eigen values of the covariance matrix.

5. Choosing component and forming feature vector.

6. Apply step 2 to 5 on captured image.

7. Matching the eigen values of stored image and captured image.

8. Final image is selected whose euclidean distance is small between the original image and Captured image.

\subsubsection{Discrete Cosine Transform}

The discrete cosine transform (DCT) [13] is closely related to the discrete fourier transform. The definition of the two dimensional DCT can be written as follows, in terms of pixel values $\mathrm{f}(\mathrm{x}, \mathrm{y})$ for $\mathrm{x}, \mathrm{y}=0,1, \mathrm{~N}-1$ and the frequency domain transform coefficients $\mathrm{F}(\mathrm{u}, \mathrm{v})$,

$$
\begin{gathered}
F(u, v)=\alpha(u) \alpha(v) \sum_{x=0}^{N-1} \sum_{y=0}^{N-1} f(x, y) \\
\cos \left(\frac{(2 x+1) u \pi}{2 N}\right) \cos \left(\frac{(2 y+1) v \pi}{2 N}\right)
\end{gathered}
$$

For $\mathrm{u}, \mathrm{v}=0,1,2, \ldots \ldots \ldots \mathrm{N}-1$ and

$$
\begin{gathered}
f(x, y)=\sum_{u=0}^{N-1} \sum_{v=0}^{N-1} \alpha(u) \alpha(v) F(u, v) \\
\cos \left(\frac{(2 x+1) u \pi}{2 N}\right) \cos \left(\frac{(2 y+1) v \pi}{2 N}\right)
\end{gathered}
$$

The N X N cosine Transform Matrix $C=\{C(u, v)\}$ is define as,

$$
\mathrm{C}(\mathrm{u}, \mathrm{v})=\left\{\begin{array}{cc}
\sqrt{1} / N & \mathrm{u}=0,0 \leq \mathrm{v} \leq \mathrm{N}-1 \\
\sqrt{2} / N & \cos [\pi(2 v+1) \mathrm{u} / 2 \mathrm{~N} \\
1 \leq \mathrm{u} \leq \mathrm{N}-1,0 \leq \mathrm{v} \leq \mathrm{N}-1
\end{array}\right.
$$

The Cosine Matrix is real and orthogonal but not symmetric,

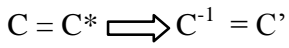

C.C' $=\mathrm{I}$

The 2D DCT of an image can be generated using equation,

$\mathrm{F}=\mathrm{C}$ f C'

If DCT is applied to real data, the result is also real. The DCT tends to concentrate information, making it useful for image compression applications and also helping in minimizing feature vector size for different applications [12]. For full 2- 
Dimensional DCT for an NXN image the number of multiplications required are $\mathrm{N}^{2}(2 \mathrm{~N})$ and number of additions required are $2 \mathrm{~N}^{2}(\mathrm{~N}-1)$.

\subsubsection{Walsh Transform}

Walsh transform matrix [13] is defined as a set of $\mathrm{N}$ rows, denoted $\mathrm{W}_{\mathrm{j}}$, for $\mathrm{j}=0,1, \ldots, \mathrm{N}-1$, which have the following properties:

- $\mathrm{W}_{\mathrm{j}}$ takes on the values +1 and -1 .

- $\mathrm{Wj}[0]=1$ for all $\mathrm{j}$.

$\cdot \mathrm{Wj} \times \mathrm{Wk}^{\mathrm{T}}=0$, for $\mathrm{j} \neq \mathrm{k}$ and $\mathrm{Wj} \times \mathrm{Wk}^{\mathrm{T}}=\mathrm{N}$, for $\mathrm{j}=\mathrm{k}$.

- $\mathrm{W}_{\mathrm{j}}$ has exactly $\mathrm{j}$ zero crossings, for $\mathrm{j}=0,1, \ldots ., \mathrm{N}-1$.

- Each row $\mathrm{W}_{\mathrm{j}}$ is even or odd with respect to its midpoint.

Walsh transform matrix is defined using a hadamard matrix of order N. The row of Walsh transform matrix is considered as the row of the hadamard matrix. It specified by the Walsh code index, which must be an integer in the range $[0, \ldots, \mathrm{N}$ 1]. For the Walsh code index equal to an integer $j$, the respective Hadamard output code has exactly j zero crossings, for $\mathrm{j}=0,1, \ldots, \mathrm{N}-1$.

For the full 2-Dimensional Walsh transform applied to image of size NXN, the number of additions required are $2 \mathrm{~N}^{2}(\mathrm{~N}-1)$ and absolutely no multiplications are needed in Walsh transform [13].

\subsubsection{Haar Transform}

The Haar Transform is deriving from Haar matrix. The Haar transform like most of the other transform is separable and can be expressed in matrix form,

$\mathrm{F}=\mathrm{H} \mathrm{f} \mathrm{H}^{\prime}$

Where $\mathrm{f}$ is an NXN image, $\mathrm{H}$ is a NXN matrix and $\mathrm{F}$ is resultant NXN transform The transform $\mathrm{H}$ contains the Haar basis function $\mathrm{h}_{\mathrm{k}}(\mathrm{x})$ which are defined over the continuous closed interval $\mathrm{x}[0,1]$

Haar basis functions are,

When, $\mathrm{k}=0$ the Haar function is defined as a constant

$$
\mathrm{h}_{0}(\mathrm{t})=1 / \sqrt{N}
$$

When, $\mathrm{k}>0$ the Haar function is defined by

$$
H_{k}(t)=1 / \sqrt{N}\left\{\begin{array}{cc}
2^{p / 2} & (q-1) / 2^{p} \leq t<(q-0.5) / 2^{p} \\
-2^{p / 2} & (q-0.5) / 2^{p} \leq t<q / 2^{p} \\
0 & \text { otherwise }
\end{array}\right.
$$

From the definition, it can be seen that $\mathrm{p}$ determines the amplitude and width of the non-zero part of the function, while $\mathrm{q}$ determines the position of the non-zero part of the function.

\subsubsection{Kekre's Transform}

Kekre's transform matrix is the generic version of Kekre's LUV color space matrix [12]. Kekre's transform matrix can be of any size NXN, which need not have to be in powers of 2 (as is the case with most of other transforms). All upper diagonal and diagonal values of Kekre's transform matrix are one, while the lower diagonal part except the values just below diagonal is zero. Generalized NXN Kekre's transform matrix can be given as:

$\left[\begin{array}{llllll}1 & 1 & \ldots & 1 & 1 & 1 \\ -\mathrm{N}+1 & 1 & \ldots & 1 & 1 & 1 \\ 0 & -\mathrm{N}+2 & \ldots . . & 1 & 1 & 1 \\ . & . & \ldots & . & . & . \\ . & . & \ldots & . & . & . \\ 0 & 0 & . & 0 & 1 & 1 \\ 0 & 0 & . & 0 & -\mathrm{N}+(\mathrm{N}-1) & 1\end{array}\right]$

The formula for generating the term Kxy of Kekre's transform matrix is:

$$
\text { Kxy }=\left\{\begin{array}{cl}
1 & ; x<=y \\
-N+(x-1) & ; x=y+1 \\
0 & ; x>y+1
\end{array}\right.
$$

For taking Kekre's transform of an NXN image, the number of required multiplications are $2 \mathrm{~N}(\mathrm{~N}-2)$ and number of additions required are $\mathrm{N}\left(\mathrm{N}^{2}+\mathrm{N}-2\right)$.

\section{PROPOSED ALGORITHM}

1. Capture the image, or take the input image from stored database.

2. Perform preprocessing on input image using boundary point selection and cropping point calculation.

3. For every column compute the mean i.e. column mean,

$$
\mathrm{C} 11=(\mathrm{X} 11+\mathrm{X} 21+\mathrm{X} 31 \ldots \ldots \mathrm{XN} 1) / \mathrm{N} \text {. }
$$

Similarly, for every row compute the mean i.e. row mean,

$\mathrm{R} 11=(\mathrm{R} 11+\mathrm{R} 12+\mathrm{R} 13---\mathrm{R} 1 \mathrm{~N}) / \mathrm{N}$.

4. Apply transformation on column mean and row mean.

5. Select column mean or row mean of different sizes to calculate feature vector as shown in Fig. 4.

6. Compare feature vector of the query image with the feature vectors of all the images in the database.

7. To calculate the closest match with query image it measures euclidean distance to check the closeness of the query image and the database images.

8. Select the smallest value with respect to the corresponding column to find first closest match with the query image.

9. Display the equivalent image from the database. 


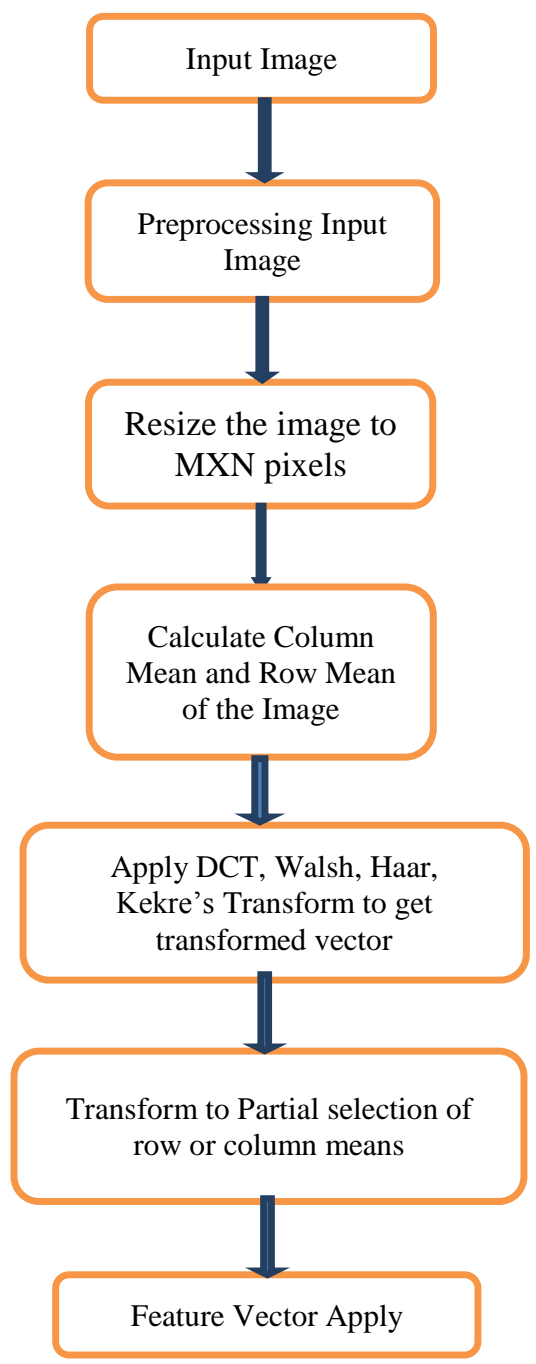

Fig. 4: Flowchart for Feature Extraction

\section{RESULT AND DISCUSSION}

The implementation of the proposed algorithm is done in MATLAB 10 using a computer with Intel Core i3 processor. The proposed algorithm is tested on 48 different gestures representing American Sign Languages [14]. The hand gestures for training were collected with the plane background by same hand gesture 5 times; each time from a different angle and position. The dataset contains $120(=24 * 5)$ images of each hand gestures. For testing $48(=24 * 2)$ images have been used.

This Database is tested using different feature extraction techniques such as principle component analysis and transform techniques. The result shows that with principle component analysis the average percentage of accuracy is 98\%. Same database is tested on the transformed techniques with various sizes of feature vectors. Table 1 shows the percentage accuracy for different transform domain method like DCT, Haar, Walsh and Kekre's.
Table 1. Percentage Accuracy with different Sizes of Transformed matrix

\begin{tabular}{|c|c|c|c|c|c|}
\hline Methods & \multicolumn{5}{|c|}{ Accuracy in \% } \\
\hline Transform & $\mathbf{1 0 0 \%}$ & $\mathbf{5 0 \%}$ & $\mathbf{2 5 \%}$ & $\mathbf{1 2 . 5 \%}$ & $\mathbf{6 . 2 5 \%}$ \\
\hline DCT & $100 \%$ & $100 \%$ & $100 \%$ & $100 \%$ & $95 \%$ \\
\hline Haar & $100 \%$ & $100 \%$ & $100 \%$ & $98 \%$ & $84 \%$ \\
\hline Walsh & $100 \%$ & $100 \%$ & $100 \%$ & $98 \%$ & $84 \%$ \\
\hline Kekre's & $88 \%$ & $84 \%$ & $75 \%$ & $55 \%$ & $46 \%$ \\
\hline
\end{tabular}

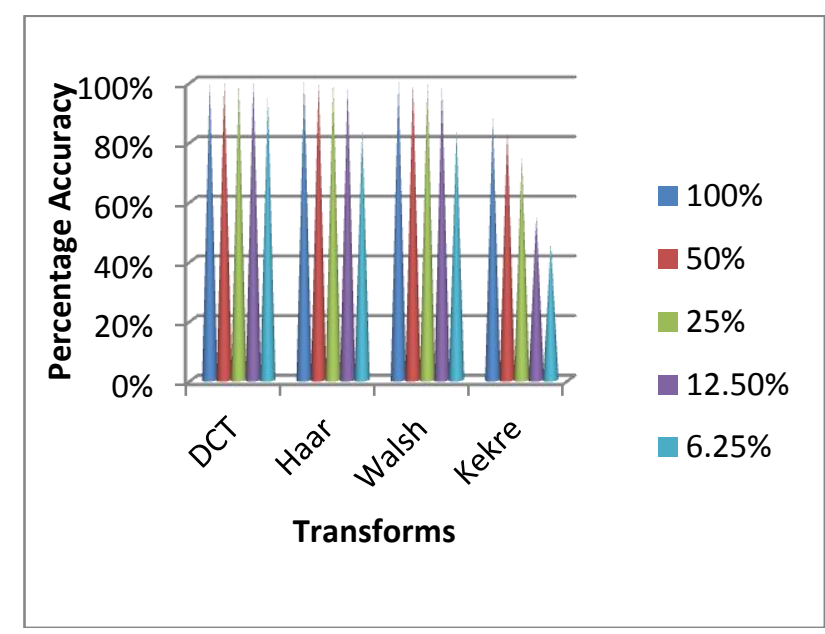

Fig. 5: Percentage Accuracy of all the transforms

The average recognition rate of the principle component analysis method is $9 \mathrm{Sec}$. whereas the recognition rate of the proposed system 0.92 Seconds Table 2 shows the computational complexity of the transforms for the image of size $\mathrm{N}$ X N.

Table 2. Computational Complexity for transforms to image of size $\mathrm{N} X \mathrm{~N}$

\begin{tabular}{|c|c|c|c|}
\hline $\begin{array}{c}\text { Transfo } \\
\text { rm }\end{array}$ & $\begin{array}{c}\text { Number of } \\
\text { Additions }\end{array}$ & $\begin{array}{c}\text { Number } \\
\text { of } \\
\text { Multipli } \\
\text { cation }\end{array}$ & $\begin{array}{c}\text { Total } \\
\text { Additions } \\
\text { for } \\
\text { transform } \\
\text { of 64 X 64 } \\
\text { image }\end{array}$ \\
\hline DCT & $2 \mathrm{~N}^{2}(\mathrm{~N}-1)$ & $\mathrm{N}^{2}(2 \mathrm{~N})$ & 4710400 \\
\hline Haar & $2 \mathrm{~N}^{2} \log _{2}(\mathrm{~N})$ & 0 & 49152 \\
\hline Walsh & $2 \mathrm{~N}^{2}(\mathrm{~N}-1)$ & 0 & 516096 \\
\hline Kekre's & $\mathrm{N}[\mathrm{N}(\mathrm{N}+1)-2]$ & $2 \mathrm{~N}(\mathrm{~N}-2)$ & 329600 \\
\hline
\end{tabular}




\section{CONCLUSION AND FUTURE WORK}

In today's digitized world, processing speed has increase dramatically, with computers being advanced to the levels where they can assist humans in complex tasks. Yet, input technologies cause a major bottleneck in performing some of the tasks, underutilizing the available resources and restricting the expressiveness of application use. Hand gesture recognition techniques is used to interact with the computer more easily and user friendly.

This proposed system is simple but effective. The various transforms like DCT, Haar, Walsh and Kekre's transform is used for gesture recognition. A column mean of each transform image and various percentages of feature vectors such as $100 \%, 50 \%, 25 \%, 12.5 \%$ which gives $100 \%$ result and accuracy compare to the existing system such as principle component analysis. The recognition speed is $0.9 \mathrm{sec}$. The complexity comparison of all the transform shows that the complexity of Haar transform is lesser than the other transforms.

Future Scope for this project will be focused on more complicated gestures and gestures with complex background.

\section{ACKNOWLEDGMENTS}

We would like to express our sincere thanks to all the staff of Department of Computer Science, Thadomal Shahani, College of Engineering, for their support and guidance throughout.

\section{REFERENCES}

[1] Jagdish Lal Raheja, Radhey Shyam, Umesh Kumar, P Bhanu Prasad, " Real-Time Robotic Hand Control using Hand gestures" proceeding of International Conference on Machine Learning and Computing (ICMLC) of IEEE, pp.12-16, Feb 2010.

[2] Yuehai Wang, JianfeiLi "Entertainment Robot Hand Gesture Recognition" proceeding of International Conference on Database Technology and applications (DBTA), pp. 27-28, Nov. 2010.

[3] Yikai Fang, Kongqiao Wang, Jian cheng,Hanqing Lu "A Real-Time Hand Gesture recognition Method" Proceeding of International conference on Multimedia and Expo. IEEE pp.995-998, July 2007

[4] Deng-Yuan Huang, Wu-Chih Hu, Sung Hsiang Chang "Vision- based Hand Gesture Recognition Using PCA+Gabor Filters and SVM" proceeding of International Conference on Intelligent information hiding and Multimedia Signal Processing, pp 1-4, Sep 12, 2009.
[5] Qing Chen, Nicolas D. Georganas, Emil M. Petriu “ Real-time Vision-based Hand Gesture Recognition Using Haar-like Features" Proceeding of international conference on Instrumentation and Measurement Technology IEEE, pp 1-6, May 1-3 2007.

[6] Rajeshree Rokade, Dharmpal Doye, Manesh Kokare "Hand Gesture Recognition by Thinning Method" International Conference on Digital Image processing 2009 IEEE pp 284-287, March 7,2009.

[7] Zhong Yang, Yi Li, Weidong Chen, Yang Zheng "Dynamic Hand Gesture Recognition Using Hidden Markov Models" Proceeding of 7th International Conference on Computer Science and Education. Melbourne, Australia IEEE July 14-17, 2012.

[8] Karishma Dixit, Anand Singh Jalal , "Automatic Indian Sign Language Recognition System" Proceeding of IEEE $3^{\text {rd }}$ international conference on Advance computing, pp. 883-887, Feb 22- 23, 2013.

[9] Archana S. Ghotkar, Rucha Khatal , Sanjana Khupase, Surbhi Asati and Mithila Hadap "Hand Gesture Recognition for Indian Sign Language" Proceeding of 3rd IEEE International Conference on Advance Computing , pp1-4, Jan 10-12, 2012.

[10] Prateem Chakraborty, Prashant Sarawgi, Gaurav agrawal, "Hand Gesture Recognition: A Comparative Study" proceeding of the International multi Conference of Engineers and Computer Scientists ISBN, pp.19-21, March 2008.

[11] Nasser H. Dardas and Emil M. Petriu "Hand Gesture Detection and Recognition Using Principal component Analysis" proceeding of International Conference on Digital Object Identifier, pp1-6, Sept 2011.

[12] H. B. Kekre, Sudeep D. Thepade and Akshay Maloo "Performance Comparison of Image Retrieval Using Fractional Coefficients of Transformed Image Using DCT, Walsh, Haar and Kekre's transform" Proceeding of International Journal of Image Processing (IJIP)

[13] H. B. Kekre, Tanuja Sarode, Meena Ugale, "Performance Comparison of Image Classifier Using Discrete Cosine Transform and Walsh Transform" Proceeding of 2nd International Conference and workshop on Emerging Trends in Technology (ICWET) 2011.

[14]Thomas Moeslund's Gesture Recognition Database, http://www-Prima.inrialpes.fr/FGnet/data/12-Moeslund Gesture/ database.html 